\title{
Study of TNF- $\alpha$, IL-1 $\beta$ and LPS levels in the gingival crevicular fluid of a rat model of diabetes mellitus and periodontitis
}

\author{
Zhu-Ling Jiang ${ }^{\mathrm{a}}$, Yu-Qiong Cui ${ }^{\mathrm{a}}$, Rui Gao ${ }^{\mathrm{b}}$, Ying $\mathrm{Li}^{\mathrm{a}}$, Zhao-Chen $\mathrm{Fu}^{\mathrm{c}}$, Bin Zhang ${ }^{\mathrm{a}, *}$ and \\ Cheng-Chao Guan ${ }^{\mathrm{a}, *}$ \\ ${ }^{a}$ Department of Implantology, the 2nd Affiliated Hospital, Harbin Medical University, Harbin, Heilongjiang, China \\ ${ }^{\mathrm{b}}$ Department of Orthodontics, the 1st Affiliated Hospital, Harbin Medical University, Harbin, Heilongjiang, China \\ ${ }^{\mathrm{c}}$ Department of Oral and Maxillofacial Surgery, the 2nd Affiliated Hospital, Harbin Medical University, Harbin, \\ Heilongjiang, China
}

\begin{abstract}
.
OBJECTIVE: In this study, we sought to investigate the dynamic changes in the levels of TNF- $\alpha$, IL- $1 \beta$ and LPS in the gingival crevicular fluid (GCF) in a rat model of diabetes mellitus (DM) and periodontitis (PD). Additionally, we evaluated alveolar bone loss and the histopathological response associated with experimental diabetes mellitus and experimental periodontitis.

METHODS: DM and PD were induced together in 15 rats (group 1) by streptozotocin injection and ligature induction. Periodontitis alone was produced by ligature induction in 15 rats (group 2), diabetes alone was produced by streptozotocin injection in 15 rats (group 3), and fifteen systemically and periodontally healthy rats were used as controls (group 4). The gingival TNF- $\alpha$, IL-1 $\beta$ and LPS levels were measured by using ELISA method. Periodontal destruction was assessed by measuring the alveolar bone loss. Periodontal inflammation was quantified by histopathological grading in H\&E stained samples.

RESULTS: Higher levels of TNF- $\alpha$, IL1- $\beta$ and LPS, increased alveolar bone loss and more serve histopathology were found in group 1 compared with group 2, group 3 and group $4(p<0.05)$. The quantities of TNF- $\alpha$, IL1- $\beta$ and LPS, the amount of alveolar bone loss and the severity of the histopathological finding were greater in group 2 than group 3 and group $4(p<0.05)$. Group 3 demonstrated higher levels of TNF- $\alpha$, IL1- $\beta$ and LPS, increased alveolar bone loss and more serve histopathology than group $4(p<0.05)$. Statistically significant differences were noted between all of the groups.

CONCLUSIONS: These data indicate that DM may lead to enhanced TNF- $\alpha$, IL1- $\beta$ and LPS production in the periodontal tissues. The resorption values of alveolar bone and the histological inflammation were more severe in rats with periodontitis and diabetes mellitus than in those with periodontitis alone, diabetes mellitus alone and control rats. Our findings are consistent with the hypothesis that hyperglycemia contributes to the heightened inflammatory response associated with periodontitis.
\end{abstract}

Keywords: TNF- $\alpha$, IL-1 $\beta$, LPS, GCF, diabetes mellitus, periodontitis, alveolar bone loss, histopathology

\section{Introduction}

Diabetes mellitus and periodontal disease are both chronic inflammatory disorders that have a major impact on the health of millions of individuals worldwide.

\footnotetext{
*Corresponding authors: Bin Zhang and Cheng-Chao Guan, Department of Implantology, the 2nd Affiliated Hospital, Harbin Medical University, Harbin 150086, Heilongjiang, China. E-mail: zhb19622003@yahoo.com.cn, dentiguan@163.com.
}

Periodontal disease is a recognized and well-documented complication of diabetes. The evidence supporting this relationship is based on epidemiologic data and animal model studies that have helped to explain the pathophysiology of periodontal disease as a complication of diabetes mellitus [1]. Periodontal disease was proposed as the sixth complication of diabetes mellitus. In 2008, Taylor and Borgnake [2] identified periodontal disease as a possible risk factor for poor metabolic control in people with diabetes melli- 
tus. This bidirectional relationship between periodontal disease and diabetes mellitus makes diabetes a disorder of importance to dentists and dental hygienists as well as dental patients.

Tumour necrosis factor (TNF- $\alpha$ ) has been reported to play a key role in the pathogenesis of type $2 \mathrm{di}-$ abetes [3]. Several lines of evidence support this hypothesis. In animal [4] and in vitro human cell models, TNF- $\alpha$ inhibits the action of insulin. In human crosssectional studies, elevated levels of circulating TNF- $\alpha$ are associated with insulin resistance [5] and type 2 diabetes [6]. Longitudinal human study shows elevated plasma TNF- $\alpha$ to be concomitant with worsening of glycemic control [7]. Recently, Bretz et al. [8] showed that circulating TNF- $\alpha$ was associated with more severe periodontitis in a large geriatric cohort. Subjects with periodontitis appear to have an elevated TNF$\alpha$ response, as lipopolysaccharide (LPS)-challenged macrophages derived from periodontitis patients release greater quantities of TNF- $\alpha$ compared with nonperiodontitis controls. Salvi et al. [9] noted that monocytes from subjects with both diabetes and periodontitis appears to release greater quantities of TNF- $\alpha$.

Meanwhile, Teles [10] compared the mean levels of IL- $1 \beta$ in GCF samples from periodontally healthy subjects and subjects with chronic periodontitis and found that IL- $1 \beta$ displayed the strongest relationship to the clinical signs of periodontitis. These findings support previous reports [11] of associations between the levels of IL- $1 \beta$ and the clinical signs of periodontal disease. Diabetes mellitus patients have elevated levels of advanced glycation end products (AGEs) [12] that can interact with specific receptors, such as macrophages, stimulating the production of cytokines such as IL1. Monocytes from diabetes mellitus patients produce significantly greater amounts of IL- $1 \beta$ in vitro than monocytes from non-diabetic controls [13].

Lipopolysaccharide is one of the most important microbial virulence factors in generating host-mediated periodontal tissue destruction. LPS provides a microbe-associated molecular pattern that is recognized by receptors on resident immune and non-immune cells within the periodontium [14]. Rogers [15] demonstrated that LPS endotoxin from the periodontal pathogen A. actinomycetemcomitans initiated severe alveolar bone loss and significant elevations in the levels of inflammatory cytokines and osteoclastogenesis. Bacterial LPS is a potent stimulator of many inflammatory mediators, including IL- $1 \beta$ and TNF- $\alpha$ [16].

It has been hypothesized that type 2 diabetes mellitus-related alterations in the levels of inflammatory mediators in the gingival crevicular fluid (GCF) levels of inflammatory mediators maybe part of the mechanism by which diabetes affects periodontal health. Therefore, the aim of this study was to evaluate GCF levels of TNF- $\alpha$, IL- $1 \beta$ and LPS in a Sprague-Dawley rat model with type $2 \mathrm{DM}$ and/or periodontal disease.

\section{Materials and methods}

\subsection{Statement of ethics}

All animal work has been conducted in accordance with international animal experimentation guidelines (NIH Pub. No. 85-23, revised 1996) and was approved by Animal Care and Use Committee of the Harbin Medical University (Harbin, China). All necessary permits were obtained for the described field studies.

\subsection{Production of experimental rats with DM}

A total of 60 male Sprague-Dawley rats (200-220 g) were obtained from the experimental animal center of the second affiliated hospital of Harbin Medical University (Harbin, China). The animals were acclimatized for at least 1 week before the start of the experiment and were housed in a temperature-controlled animal facility under a 12-hour light/dark cycle with enough laboratory chow and water. The experimental protocols were carried out following ethical principles for laboratory animal use. A total of 30 rats were fed an adequate HF diet [17] (34.5\% fat, $17.5 \%$ protein, $48 \%$ carbohydrate; Beijing HFK Bio-Technology, China), and the other 30 rats were received normal chow for four weeks. Then the rats that fed with HF diet were injected under low light with a single intraperitoneal injection of freshly prepared streptozocin [17] (STZ, $30 \mathrm{mg} / \mathrm{kg}$; i.p. in $0.1 \mathrm{~mol} / \mathrm{L}$ citrate buffer, $\mathrm{pH} 4.5$; Sigma Aldrich, Co., 3050 Spruce Street, St. Louis, MO 63103 USA) to induce diabetes models after abrosiaed for at least 12 hours. The blood glucose levels were measured randomly on the third and seventh days after STZ injection with a commercial blood glucose monitoring kit in blood samples obtained from the tail vein. Rats with $\mathrm{RBG}>11.1 \mathrm{mmol} / \mathrm{L}$ in two consecutive analyses were considered diabetic [18]. DM was confirmed by the increase in the blood glucose level and the body weight loss as described in the referred studies. 


\subsection{Production of experimental rats with $P D$}

A total of 15 systemically healthy rats were anesthetized by a single intraperitoneal injection of $10 \%$ chloral hydrate (Traditional Chinese Medicine Group Chemical Reagent Co., Ltd. No: 20080325) at a dose of $0.3 \mathrm{ml}$ per $100 \mathrm{~g}$ of body weight [19]. After the anesthesia, a 1-0 cotton ligature (Ethicon, Johnson and Johnson, São Paulo, SP, Brazil) was placed around the maxillary second molar in a submarginal position to induce experimental periodontitis [20,21]. The ligature was knotted on the buccal side of the teeth. The ligatures were left in place until the collection of GCF. A new ligature was placed immediately after the collection of GCF. Periodontitis was confirmed by the grade of the infiltration of inflammatory cells in the gingival histopathological analysis and alveolar bone loss quantification at the experimental sites.

\subsection{Production of experimental rats with DM and PD}

A total of 15 diabetic rats underwent the procedures described above to induce periodontitis and diabetes mellitus.

The groups were as follows:

Group 1: rats with diabetes mellitus and periodontitis $(n=15)$

Group 2: systemically healthy rats with periodontitis $(n=15)$

Group 3: periodontally healthy rats with diabetes mellitus $(n=15)$

Group 4: systemically and periodontally healthy rats $(n=15)$

Gingival crevicular fluid (GCF) collection

Glucose concentrations and body weight were measured once a week in all 60 rats. Five rats were randomly selected from each group for the collection of gingival crevicular fluid (GCF) from the mesio-buccal region of the maxillary right molar under anesthesia as previously described [22,23]. GCF was collected during the second, fourth and sixth weeks after induction of periodontitis. The teeth were air dried and isolated with cotton rolls after removing the ligament. Without touching the marginal gingiva, GCF was collected using adsorbent paper points (Beijing, Dayading Medical Apparatus and Instruments Co., Ltd.). The GCF collection was repeated three times per site, and seven absorbent paper points were used for each collection. The last paper point used for each sampling was held in the gingival sulcus for 30 seconds. After collection, the paper points were immediately transferred into a plastic vial with $20 \mu \mathrm{l}$ sample diluent (sample diluent from Rat TNF- $\alpha$ Platinum ELISA KIT, sample diluent from Rat IL- $1 \beta$ ELISA KIT, sample diluent from Rat LPS ELISA KIT) and stored at $-80^{\circ} \mathrm{C}$ until the assay.

\subsection{Analysis of TNF- $\alpha, I L-1 \beta$ and LPS in the GCF}

The TNF- $\alpha$, IL- $1 \beta$ and LPS levels in the GCF from each group were measured using an enzyme-linked immunosorbent assay (ELISA). This assay was a sandwich ELISA and was performed according to manufacturer's instructions. All of the samples and standards were assayed in duplicate, as suggested by the manufacturer (Rat TNF- $\alpha$ ELISA KIT eBioscience Campus Vienna Biocenter2 1030 Vienna, Austria; Rat IL$1 \beta$ ELISA KIT NeoBioscience Technology Co., Ltd.; Rat LPS ELISA KIT Biovalue Technology Co., Ltd.). After appropriate dilution of the samples, $100 \mu \mathrm{L}$ of each sample was added to the appropriate wells, and the plate was sealed and incubated at $37^{\circ} \mathrm{C}$ for $1 \mathrm{~h}$. Following incubation and washing seven times, $100 \mu \mathrm{L}$ of the labeled antibody was pipetted into the wells, followed by incubation at $4^{\circ} \mathrm{C}$ for $30 \mathrm{~min}$. Then, the plate was washed nine times, and $100 \mu \mathrm{L}$ of the substrate solution was added to each well followed by incubation at room temperature for $30 \mathrm{~min}$ in the dark. Lastly, $100 \mu \mathrm{L}$ of stop solution was added to each well, and the plate was read immediately to determine the absorbance of each well using a microplate (enzymelinked immunosorbent assay) reader (Thermo Labsystems Multiskan Mk 3) set at a wavelength of $450 \mathrm{~nm}$ (OD450). The levels of IL-1 $\beta$, TNF- $\alpha$ and LPS in the tested samples were computed using the standard curve plotted with the optical density values obtained from the assay.

\subsection{Assessment of alveolar bone loss}

The rats were sacrificed with an overdose of sodium pentobarbital the same day after the GCF was collected, and the excised maxillae were fixed in $10 \%$ neutral buffered formalin for 48 hours. Both maxillary halves were fixed. Then, one lateral maxillary half was used for measurement of alveolar bone loss. The evaluation of the extent of periodontal destruction based on the measurement of the alveolar bone loss was performed as described previously [24]. Quantification was performed by a investigator who was unaware of the samples analyzed through measuring the distance between the $\mathrm{CEJ}$ and the $\mathrm{BC}$ along the axis of each root 
Table 1

The concentrarions (mean $\pm \mathrm{SD}, \mathrm{pg} / \mathrm{ml}$ ) of TNF- $\alpha$ in samples collected at each time point in different groups throughout the study

\begin{tabular}{llll}
\hline Groups & Week 2 & Week 4 & Week 6 \\
\hline Diabetes and periodontitis & $35.59 \pm 2.80^{\mathrm{d}}$ & $68.49 \pm 1.20^{\mathrm{d}, *}$ & $48.88 \pm 3.65^{\mathrm{d}, *, \#}$ \\
Periodontitis & $29.46 \pm 0.69^{\mathrm{c}}$ & $57.76 \pm 0.88^{\mathrm{c}, *}$ & $33.87 \pm 2.00^{\mathrm{c}, *, \#}$ \\
Diabetes & $26.02 \pm 1.33^{\mathrm{b}}$ & $41.52 \pm 4.21^{\mathrm{b}, *}$ & $28.92 \pm 2.06^{\mathrm{b}, *, \#}$ \\
Normal & $16.38 \pm 3.77^{\mathrm{a}}$ & $19.38 \pm 2.75^{\mathrm{a}}$ & $14.53 \pm 2.32^{\mathrm{a}}$ \\
\hline
\end{tabular}

Data are means \pm SD. Data with different superscript letters are significantly different $(P<0.05)$ in the same time among four groups. ${ }^{*}$ Different from week $2(P<0.05)$. \# Different from week $4(P<0.05)$.

Table 2

The concentrarions (mean $\pm \mathrm{SD}, \mathrm{pg} / \mathrm{ml}$ ) of IL- $1 \beta$ in samples collected at each time point in different groups throughout the study

\begin{tabular}{lrrr}
\hline Groups & Week 2 & Week 4 & Week 6 \\
\hline Diabetes and periodontitis & $121.82 \pm 2.42^{\mathrm{d}}$ & $145.10 \pm 3.48^{\mathrm{d}, *}$ & $196.89 \pm 4.52^{\mathrm{d}, *, \#}$ \\
Periodontitis & $86.54 \pm 3.20^{\mathrm{c}}$ & $90.84 \pm 4.63^{\mathrm{c}}$ & $92.92 \pm 3.07^{\mathrm{c}}$ \\
Diabetes & $32.86 \pm 3.92^{\mathrm{b}}$ & $32.67 \pm 4.76^{\mathrm{b}}$ & $36.22 \pm 2.07^{\mathrm{b}}$ \\
Normal & $21.21 \pm 2.34^{\mathrm{a}}$ & $19.38 \pm 4.09^{\mathrm{a}}$ & $18.31 \pm 2.08^{\mathrm{a}}$ \\
\hline
\end{tabular}

Data are means \pm SD. Data with different superscript letters are significantly different $(P<0.05)$ in the same time among four groups. ${ }^{*}$ Different from week $2(P<0.05)$. \# Different from week $4(P<0.05)$.

Table 3

The concentrarions (mean $\pm \mathrm{SD}$, eu/ml) of LPS in samples collected at each time point in different groups throughout the study

\begin{tabular}{llll}
\hline Groups & Week 2 & Week 4 & Week 6 \\
\hline Diabetes and periodontitis & $0.572 \pm 0.034^{\mathrm{d}}$ & $0.701 \pm 0.047^{\mathrm{d}, *}$ & $0.662 \pm 0.051^{\mathrm{d}, *}$ \\
Periodontitis & $0.368 \pm 0.034^{\mathrm{c}}$ & $0.432 \pm 0.025^{\mathrm{c}, *}$ & $0.412 \pm 0.013^{\mathrm{c}, *}$ \\
Diabetes & $0.163 \pm 0.009^{\mathrm{b}}$ & $0.225 \pm 0.011^{\mathrm{b}, *}$ & $0.194 \pm 0.014^{\mathrm{b}, *}$ \\
Normal & $0.081 \pm 0.003^{\mathrm{a}}$ & $0.086 \pm 0.005^{\mathrm{a}}$ & $0.087 \pm 0.005^{\mathrm{a}}$ \\
\hline
\end{tabular}

Data are means \pm SD. Data with different superscript letters are significantly different $(P<0.05)$ in the same time among four groups. ${ }^{*}$ Different from week $2(P<0.05)$.

Table 4

Values of alveolar bone absorption (mean $\pm \mathrm{SD}, \mathrm{mm})$ of rats

\begin{tabular}{lccc}
\hline Groups & Week 2 & Week 4 & Week 6 \\
\hline Diabetes and periodontitis & $2.55 \pm 0.20^{\mathrm{d}}$ & $2.65 \pm 0.15^{\mathrm{d}}$ & $2.60 \pm 0.25^{\mathrm{d}}$ \\
Periodontitis & $1.65 \pm 0.15^{\mathrm{c}}$ & $1.75 \pm 0.10^{\mathrm{c}}$ & $1.80 \pm 0.10^{\mathrm{c}}$ \\
Diabetes & $1.20 \pm 0.05^{\mathrm{b}}$ & $1.30 \pm 0.20^{\mathrm{b}}$ & $1.30 \pm 0.05^{\mathrm{b}}$ \\
Normal & $0.95 \pm 0.05^{\mathrm{a}}$ & $0.90 \pm 0.10^{\mathrm{a}}$ & $0.95 \pm 0.10^{\mathrm{a}}$ \\
\hline
\end{tabular}

Data are means \pm SD. Data with different superscript letters are significantly different $(P<0.05)$ in the same period among four groups.

surface of the second molar teeth. Two recordings for two roots were made, and the total alveolar bone loss was obtained by taking the sum of the recordings from the buccal tooth surface [25].

\subsection{Histopathological analysis}

The other lateral half of maxillary was demineralized in $10 \%$ EDTA after fixation for histopathological analysis. The specimens in EDTA were demineralized for no less than 30 days. They were then dehydrated and embedded in paraffin, and paraffin serial sections $(6 \mu \mathrm{m})$ were obtained in the mesiodistal direction and stained with hematoxylin and eosin (H\&E). The histological features where a ligature had been placed were evaluated under a light microscope. Parameters such as the inflammatory cell influx and the periodontal tissue integrity were analyzed by a histologist in a single-blind fashion [26]. The grades were as follows: Score 0: the absence of or only discrete cellular infiltration (inflammatory cell infiltration was sparse and restricted to the marginal gingival region), preserved alveolar process and cementum. Score 1: moderate cellular infiltration (inflammatory cellular infiltration was present throughout the inserted gingiva), minor alveolar process resorption and intact cementum. Score 2: accentuated cellular infiltration (inflammatory cellular infiltration was present in both the gingiva and the periodontal ligament), accentuated degradation of the alveolar process, and partial destruction of the cementum. Score 3: accentuated cellular infiltrate, complete re- 

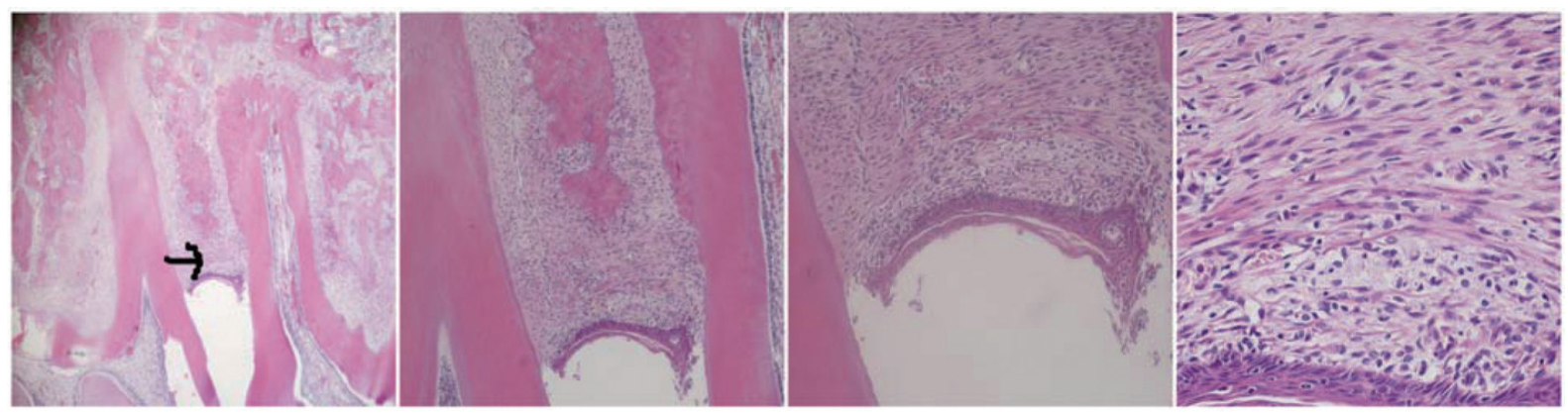

Fig. 1. Illustrates the group with STZ-induced diabetes and ligature-induced periodontitis. The histopathology of the periodontium of the animals subjected to experimental diabetes and periodontitis revealed severe inflammatory cell infiltration coupled with cementum destruction and alveolar process destruction. (Colours are visible in the online version of the article; http://dx.doi.org/10.3233/DMA-130974)

sorption of the alveolar process and severe destruction of the cementum [27].

\subsection{Statistical analysis}

The data was expressed as the mean \pm standard deviation (SD). A test for normality was performed on the data to meet the normal distribution before the twoway factorial ANOVA. When interaction and/or the main effects were significant, means were compared using the SNK post hoc test. Kruskal-Wallis test was used to test for differences in pathological grading of the periodontium among four groups. Statistical analysis was performed by using SPSS version 15.0.1 for Windows (SPSS Inc., USA). A p value less than 0.05 was considered statistically significant.

\section{Results}

\subsection{TNF- $\alpha, I L-1 \beta$ and LPS levels in $G C F$}

We sought to determine whether type 2 diabetes mellitus alters the TNF- $\alpha$ levels in GCF. The TNF- $\alpha$ levels of the rats in this study (mean \pm SD) are provided in Table 1 . The samples were analyzed individually, and the data was pooled for each rat. The TNF- $\alpha$ levels were significantly different among four groups at the same time point. Rats with periodontitis (PD) and diabetes (DM) had significantly higher levels of TNF$\alpha$ in the GCF than those with PD alone $(p<0.05)$, $\mathrm{DM}$ alone $(p<0.05)$ or the control rats $(p<0.05)$. Animals subjected to PD alone had higher levels than those with DM alone $(p<0.05)$ or controls $(p<0.05)$. Additionally, the DM rats had higher levels compared to the control rats $(p<0.05)$. While between the times, the levels of TNF- $\alpha$ were increased at week 4 and week
6 compared to week $2(p<0.05)$, and the differences were significant between week 4 and week 6 except for normal group.

To detect if type 2 diabetes mellitus affect the IL$1 \beta$ levels in the GCF. The results are provided in Table 2 and showed that IL- $1 \beta$ levels were significantly different among the four groups at the same time point. Rats with PD and DM had significantly higher levels of GCF IL- $1 \beta$ than those with PD alone $(p<0.05)$, DM alone $(p<0.05)$ and control rats $(p<0.05)$. Animals subjected to PD alone had higher levels than those with DM alone $(p<0.05)$ and control rats $(p<0.05)$. And that the DM rats had higher levels compared to control rats $(p<0.05)$. Between the different periods, only rats with PD and DM showed higher levels of IL-1 $\beta$ at week 4 and week 6 contrast to week $2(p<0.05)$. The increased level could be found between week 4 and week $6(p<0.05)$.

The LPS levels in GCF of the rats included in this study (mean \pm SD) are provided in Table 3 . We found that the levels were significantly different among the four groups at the same time point. Rats with PD and DM showed higher levels of GCF LPS than those with PD alone $(p<0.05)$, DM alone $(p<0.05)$ and control rats $(p<0.05)$. Rats subjected to $\mathrm{PD}$ alone had higher levels than those with DM alone $(p<0.05)$ and control rats $(p<0.05)$. Additionally, the DM rats had higher levels compared to the control rats $(p<0.05)$. Rats in PD and DM group, PD group and DM group expressed high levels of LPS at week 4 and week 6 compared with week $2(p<0.05)$.

\subsection{Macroscopic analysis}

Table 4 shows the resorption values of the alveolar bone in the upper second molar in the four groups at diffident time points. The rats with PD and DM showed 

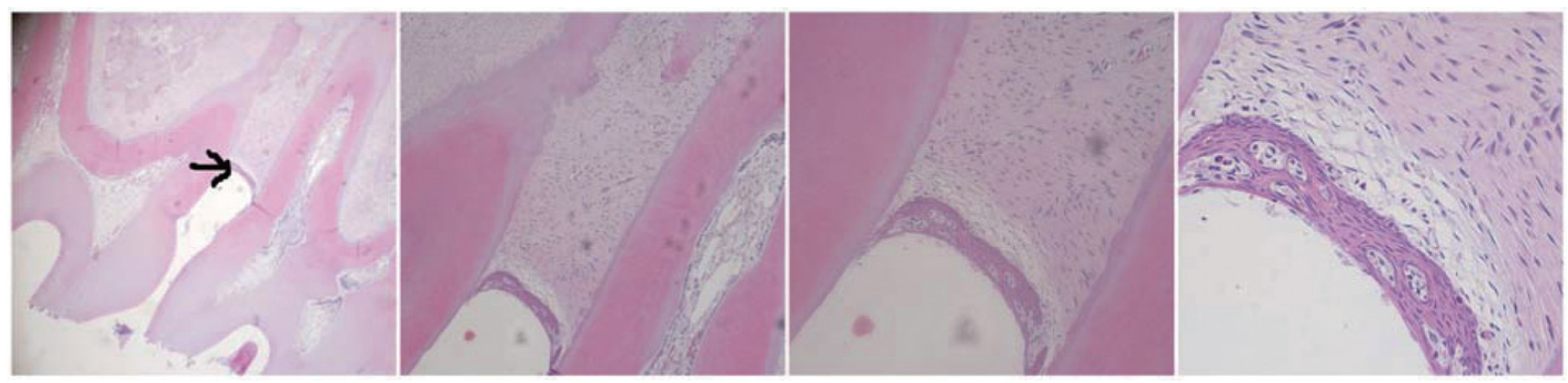

Fig. 2. Illustrates the group with ligature-induced periodontitis. Rats subjected to periodontitis revealed inflammatory cell infiltration accompanied by cementum and alveolar bone destruction. (Colours are visible in the online version of the article; http://dx.doi.org/10.3233/DMA-130974)
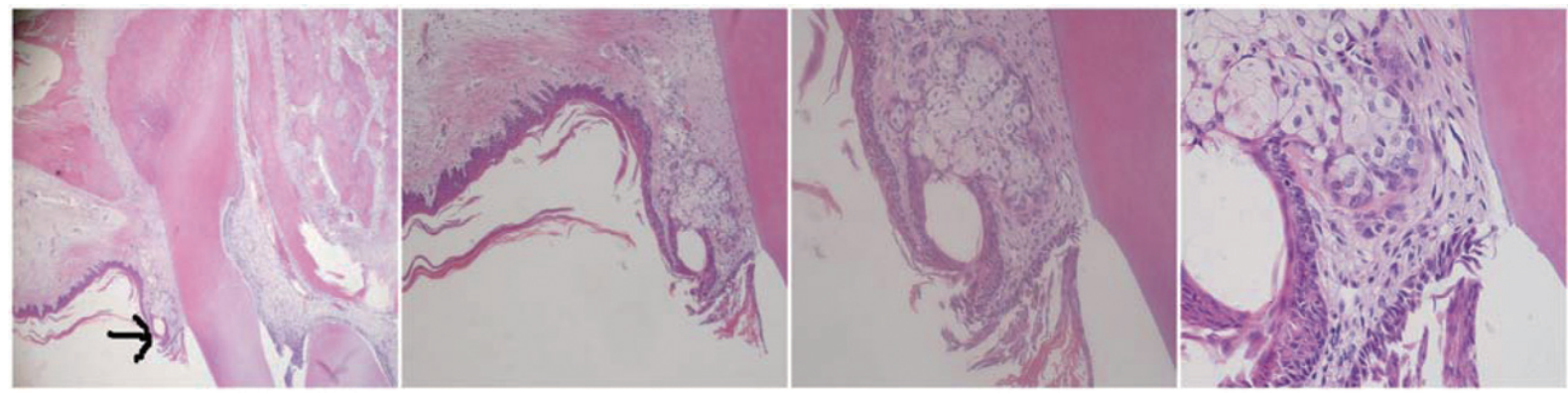

Fig. 3. Illustrates the group with STZ-induced diabetes. Rats subjected to experimental diabetes alone did not manifest bone loss, but they did exhibit inflammatory changes in the periodontium. (Colours are visible in the online version of the article; http://dx.doi.org/10.3233/DMA-130974)
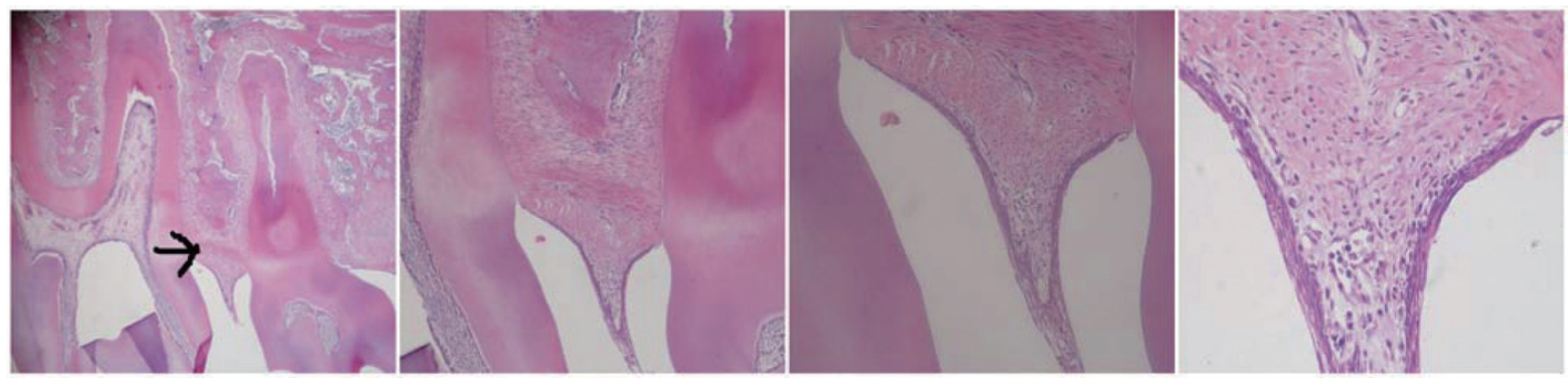

Fig. 4. Illustrates the control group. The histological analysis of group 4 shows the structure of a normal periodontium in which the gingiva, periodontal ligament, alveolar bone and cementum can be observed. (Colours are visible in the online version of the article; http://dx.doi. org/10.3233/DMA-130974)

Table 5

Pathological grading of the periodontium of rats

\begin{tabular}{lccc}
\hline Groups & Week 2 & Week 4 & Week 6 \\
\hline Diabetes and periodontitis & $2,2,2,3,2$ & $3,3,3,3,2$ & $3,3,3,3,3$ \\
Periodontitis & $1,1,1,0,1$ & $2,2,2,1,2$ & $3,3,3,2,3$ \\
Diabetes & $0,0,0,0,0$ & $1,1,1,0,1$ & $1,1,1,1,1$ \\
Normal & $0,0,0,0,0$ & $0,0,0,0,0$ & $0,0,0,0,0$ \\
\hline
\end{tabular}

Data indicates the pathological grading scores of five rats at different time periods among four groups.

more alveolar bone loss than those with PD alone, DM alone and control rats at the same time point. Rats with PD and DM had significantly greater resorption values of the alveolar bone than those with PD alone $(p<$ $0.05)$, DM alone $(p<0.05)$ and control rats $(p<$ $0.05)$. Animals subjected to PD alone had greater values than those with DM alone $(p<0.05)$ and control rats $(p<0.05)$. Additionally, the DM rats had higher values compared to the control rats $(p<0.05)$.

\subsection{Histological analysis}

Rats subjected to experimental diabetic mellitus and periodontitis exhibited inflammatory cell infiltration coupled with serve cementum destruction and alveolar 
process destruction (Fig. 1), receiving scores ranging from 2 to 3 (Table 5). The periodontitis group revealed inflammatory cell infiltration accompanied by cementum and alveolar bone destruction (Fig. 2), receiving scores ranging from 1 to 3 (Table 5). The diabetes mellitus group showed moderate or an absence of cellular infiltration, no or minor alveolar process resorption and intact cementum (Fig. 3), receiving scores ranging from 0 to 1 (Table 5). The control group presented a normal periodontium in which the gingiva, periodontal ligament, alveolar bone and cementum could be observed (Fig. 4).

\section{Discussion}

The key findings of the present study was that rats with PD and DM had high levels of TNF- $\alpha$, IL- $1 \beta$ and LPS in GCF as well as substantial resorption of the alveolar bone and histological inflammation. The gingival crevicular fluid is a serum transudate that is enriched with microbial and host products that arise as a result of the current inflammatory dynamics of the host-biofilm interaction. GCF is derived from the periodontal tissues, and its analysis provides an early indication of biochemical changes in the tissues that will ultimately manifest as a clinical lesion. Another distinct advantage of using GCF analysis as the basis for a diagnostic test is that the most popular collection system (precut methylcellulose filter strips) allows multiple sites in the mouth to be sampled and analyzed [28].

We chose to evaluate IL- $1 \beta$, LPS, and TNF- $\alpha$ because their expression occurs in periodontitis along with the loss of connective tissue and bone. The proinflammatory cytokines IL- 1 and TNF- $\alpha$ can induce bone resorption indirectly by promoting differentiation of osteoclast precursors and subsequently by activating osteoclasts. Earlier studies in models of experimental gingivitis have demonstrated the increase in GCF levels of IL-1 $\beta$ [23]. Heasman [29] conducted a study to determine the sequence of cytokine and lipid mediator activation within the periodontium during the development of experimental gingivitis in humans. They found that the GCF levels of IL- $1 \beta$ increased abruptly from mean baseline values of $16.5 \pm 9.3 \mathrm{ng} / \mathrm{ml}$ to $131 \pm 76.0 \mathrm{ng} / \mathrm{ml}$ at 1 week and remained at this level for the duration of the experiment. The findings described above are similar to our results in which rats with PD had higher levels of IL- $1 \beta$ in the GCF than control animals. Because Gram-negative microorganisms predominate in the subgingival environment and contain LPS in their outer membranes, these bacteria may be released during infection. Furthermore, it has been demonstrated that LPS elicits TNF production [30]. The priming of polymorphonuclear cells (PMNs) by LPS and TNF- $\alpha$ results in an enhanced oxidative burst and enhanced degranulation [31]. The secreted agents also enhance the production of numerous proinflammatory cytokines that contribute to the disease, including TNF- $\alpha$ and IL- $1 \beta$, among a broad array of biomolecules that have consistently been reported to be elevated in the gingival crevicular fluid (GCF) and tissues of periodontitis patients [32]. IL- $1 \beta$ and TNF$\alpha$ have been shown to dramatically enhance the oxidative burst of PMN in response to secondary stimulation [30]. These findings are accordance with our results and account for the elevated levels of TNF- $\alpha$, IL$1 \beta$ and LPS in the GCF of periodontitis patients.

Because all individuals are not equally susceptible to the destructive effects of periodontal infections, periodontitis is not only caused by bacterial infection but also associated with host susceptibility. Several biologically plausible mechanisms have been proposed to explain the interactions between diabetes and periodontal diseases. Chronic gram-negative periodontal infections may induce or perpetuate an elevated chronic systemic inflammatory state, contributing to increased insulin resistance and poor glycemic control [16]. Increased serum concentrations of acute-phase response markers and cytokines have been observed in patients with diabetes [33], which indicates that circulating inflammatory cytokines influence the risk for diabetes. The impact of glycemic control in diabetes on periodontal diseases and the mechanisms through which this effect might occur is less clear. In our studies, we found that rats with PD and DM had higher levels of TNF- $\alpha$, IL- $1 \beta$ and LPS in their GCF than animals with PD alone; meanwhile, the levels of resorption of the alveolar bone were higher and histological inflammation was more severe than in rats with PD alone. To a certain extent, our results confirm previous findings that periodontitis was more severe in subjects with diabetes compared with non-diabetics [34]. Our finding that higher levels of TNF- $\alpha$, IL- $1 \beta$ and LPS were present in the GCF from the PD and DM group than the PD group indicated that diabetes influences the gingival cytokine and LPS levels in periodontitis and worsens the macroscopic and histopathological characteristics of periodontitis.

The levels of TNF- $\alpha$, IL- $1 \beta$ and LPS were increased at week 4 and week 6 compared to week 2 . These could be due to the inflammation development process. From 
the results we found that TNF- $\alpha$ levels decreased at week 6 compared to week 4 , but still remaining significantly above week 2 . As periodontal tissue itself could make repair response to injury and the lower levels of TNF- $\alpha$ seem to be related to the preservation of homeostasis and to the remodeling/healing of damaged tissue through the activation and formation of fibroblasts [35]. Since there was no significantly increased IL-1 $\beta$ at week 4 and week 6 compared to week 2 in PD group and DM group, diabetes or periodontitis alone may not be sufficient to elicit marked changes in IL- $1 \beta$ levels over a short time. And further research is needed to study the influence of time on the gradual secrete process of cytokines.

Alveolar bone resorption is an inevitable result of periodontitis, and it has also been observed in diabetic conditions [36]. The mechanisms of alveolar bone metabolism and/or resorption in DM have been explained previously. Mishima et al. [37] and Villarino et al. [38] suggested that streptozotocin-induced DM might have had short-term effects on bone metabolism and may have reduced the rate of bone turnover in the alveolar wall surrounding the root. These are in accordance with our findings that we observed enhanced alveolar bone resorption in the DM and PD group compared with the other groups.

The main pathogenic component of bacterial biofilms is LPS, and these films have been shown to be necessary for the initiation of gingival inflammation and subsequent destruction of periodontal tissues. Based on an established model of pathogenesis, the presence of a bacterial biofilm alone is insufficient to explain disease initiation and progression. Evidence [37] suggests that periodontal tissue destruction is mainly due to the host's inflammatory response to the bacterial challenge. Investigations into the mechanisms of the host-mediated response in periodontal disease have revealed that it involves the activation of the broad axis of innate immunity, specifically by upregulation of proinflammatory cytokines (TNF- $\alpha$, IL-1 $\beta$ and so on) from monocytes and polymorphonuclear leukocytes. Toll-like receptors are the most studied pattern recognition receptors, and they are present at the cell surface as transmembrane receptors. Toll-like receptor-4 recognizes lipopolysaccharide on macrophages. Binding to the pattern recognition receptors activates the nuclear factor- $\kappa \mathrm{B}(\mathrm{NF}-$ $\kappa \mathrm{B})$ signaling pathway, which induces immune response genes, especially those for inflammatory cytokines. Inappropriate secretion of these cytokines, in terms of either type or quantity, characterizes a dys- regulated immune response that leads to destruction of the periodontal tissues in the presence of a Gramnegative bacterial biofilm. These locally produced cytokines move into the systemic circulation, where they may perpetuate an elevated inflammatory state, worsening the patient's diabetes by increasing insulin resistance and glucose levels [39]. These are the mechanisms through which periodontal infections contribute to diabetes mellitus.

In subjects with diabetes, chronically elevated blood glucose levels lead to the accelerated formation of advanced glycation end products (AGEs). Endothelial cells and monocytes possess specific receptors for AGEs located on their cell surfaces. The interaction of macrophages with AGEs has been shown to stimulate increased secretion of pro-inflammatory mediators, such as TNF- $\alpha$ and IL- $1 \beta$ [40]. As demonstrated by a 2.9-fold increase in the risk for periodontitis in individuals with poorly controlled glycemia compared with normal individuals, diabetes mellitus is a major risk factor for the development of periodontitis. However, the mechanisms by which hyperglycemia can induce periodontal destruction have not to be fully elucidated. The results of our studies revealed elevated levels of TNF- $\alpha$, IL- $1 \beta$ and LPS in the GCF of rats with $\mathrm{PD}$ and DM compared to rats with PD alone, rats with $\mathrm{DM}$ alone and control rats. The resorption values of the alveolar bone and histological inflammation were the lowest in the control rats, higher in the DM alone and PD alone groups and highest in the PD and DM group. These results might indicate that diabetes mellitus promotes periodontitis through elevated inflammatory cytokines (TNF- $\alpha$ and IL-1 $\beta$ ) and bacterial endotoxin (LPS).

Periodontitis worsens the manifestations of diabetes through the LPS-TLR4-NF- $\kappa \mathrm{B}$ signaling pathway. Toll-like receptors (TLRs) are a family of pattern recognition receptors (PRRs) that recognize microbial components and mediate the activation of host response [41]. After Toll-like receptor-4 recognizes lipopolysaccharide on macrophages, they bind then microbial LPS utilizes TLR4 to activate nuclear factor$\kappa \mathrm{B}(\mathrm{NF}-\kappa \mathrm{B})$, leading to inappropriate secretion of proinflammatory cytokines. These locally produced cytokines move into the systemic circulation increasing insulin resistance and glucose levels [39]. According to our results, it is unclear whether diabetes mellitus promotes periodontitis through the LPS-TLR4$\mathrm{NF} \kappa \mathrm{B}$ signaling pathway as well. Whether treatment based on inhibiting a part of this pathway may cure diabetes mellitus or periodontitis remains to be determined. Therefore, additional studies are warranted. 


\section{Acknowledgements}

The authors thank Nai-qian Wang (Department of Pathology, Harbin Medical University) for his excellent technical assistance.

\section{Funding}

This work was supported by Natural Science Foundation of China (No. 81170960). The funders supported study design, data collection and analysis, decision to publish, and preparation of the manuscript.

\section{Conflict of interest}

The authors declare that there is no duality of interest associated with this manuscript.

\section{References}

[1] Pontes Andersen CC, Flyvbjerg A, Buschard K, Holmstrup P, Relationship between periodontitis and diabetes: lessons from rodent studies, J Periodontol (2007), 78: 1264-1275.

[2] Taylor GW, Borgnakke WS, Periodontal disease: Associations with diabetes, glycemic control and complications, Oral Dis (2008), 14: 191-203.

[3] Nishimura F, Iwamoto Y, Mineshiba J, Shimizu A, Soga Y, Murayama Y, Periodontal disease and diabetes mellitus: The role of tumor necrosis factor-alpha in a 2-way relationship, $J$ Periodontol (2003), 74: 97-102.

[4] Hotamisligil GS, Peraldi P, Budavari A, Ellis R, White MF, Spiegelman BM, IRS-1-mediated inhibition of insulin receptor tyrosine kinase activity in TNF-alpha- and obesity-induced insulin resistance, Science (1996), 271: 665-668.

[5] Mishima Y, Kuyama A, Tada A, Takahashi K, Ishioka T, Kibata M, Relationship between serum tumor necrosis factoralpha and insulin resistance in obese men with Type 2 diabetes mellitus, Diabetes Res Clin Pract (2001), 52: 119-123.

[6] Fernandez-Real JM, Lainez B, Vendrell J, Rigla M, Castro A, Peñarroja G, Broch M, Pérez A, Richart C, Engel P, Ricart $\mathrm{W}$, Shedding of TNF-alpha receptors, blood pressure, and insulin sensitivity in type 2 diabetes mellitus, Am J Physiol Endocrinol Metab (2002), 282: E952-959.

[7] Lechleitner M, Herold M, Dzien-Bischinger C, Hoppichler F, Dzien A, Tumour necrosis factor-alpha plasma levels in elderly patients with Type 2 diabetes mellitus-observations over 2 years, Diabet Med (2002), 19: 949-953.

[8] Bretz WA, Weyant RJ, Corby PM, Ren D, Weissfeld L, Kritchevsky SB, Harris T, Kurella M, Satterfield S, Visser $M$, Newman AB, Systemic inflammatory markers, periodontal diseases, and periodontal infections in an elderly population, J Am Geriatr Soc (2005), 53: 1532-1537.

[9] Salvi GE, Beck JD, Offenbacher S, PGE2, IL-1 beta, and TNF-alpha responses in diabetics as modifiers of periodontal disease expression, Ann Periodontol (1998), 3: 40-50.
[10] Teles R, Sakellari D, Teles F, Konstantinidis A, Kent R, Socransky S, Haffajee A, Relationships among gingival crevicular fluid biomarkers, clinical parameters of periodontal disease, and the subgingival microbiota, J Periodontol (2010), 81: 89-98.

[11] Cetinkaya B, Guzeldemir E, Ogus E, Bulut S, Pro- and Antiinflammatory Cytokines in Gingival Crevicular Fluid and Serum of Rheumatoid Arthritis and Chronic Periodontitis Patients, J Periodontol 2012 Mar 13. [Epub ahead of print].

[12] Ryan ME, Carnu O, Kamer A, The influence of diabetes on the periodontal tissues, J Am Dent Assoc (2003), 134: 34S$40 \mathrm{~S}$.

[13] Salvi GE, Collins JG, Yalda B, Arnold RR, Lang NP, Offenbacher S, Monocytic TNF alpha secretion patterns in IDDM patients with periodontal diseases, J Clin Periodontol (1997), 24: 8-16.

[14] Akira S, Hemmi H, Recognition of pathogen-associated molecular patterns by TLR family, Immunol Lett (2003), 85: 85-95.

[15] Jill E. Rogers, Fei Li, Derek D. Coatney, Actinobacillus actinomycetemcomitans Lipopolysaccharide-Mediated Experimental Bone Loss Model for Aggressive Periodontitis, $\mathrm{J} \mathrm{Pe}$ riodontol (2007), 78: 550-558.

[16] Hersh D, Weiss J, Zychlinsky A, How bacteria initiate inflammation: Aspects of the emerging story, Curr Opin Microbiol (1998), 1: 43-48.

[17] K. Srinivasan, B. Viswanad, Lydia Asrat, C.L. Kaul, P. Ramarao, Combination of high-fat diet-fed and low-dose streptozotocin-treated rat: A model for type 2 diabetes and pharmacological screening, Pharmacological Research (2005), 52: 313-320.

[18] Ti Y, Xie GL, Wang ZH, Bi XL, Ding WY, Wang J, Jiang GH, Bu PL, Zhang Y, Zhong M, Zhang W, TRB3 gene silencing alleviates diabetic cardiomyopathy in a type 2 diabetic rat model, Diabetes (2011), 60: 2963-2974.

[19] Semenoff Segundo A, Semenoff TA, Borges AH, Pedro FL, Sakai VT, Methodological model of chronic stress associated with ligature-induced periodontitis in rats: a radiographic study, Braz Oral Res (2010), 24: 455-459.

[20] Semenoff TADV, Semenoff-Segundo A, Bosco AF, Nagata MJ, Garcia VG, Biasoli ER, Histometric analysis of ligatureinduced periodontitis in rats: A comparison of histological section planes, J Appl Oral Sci (2008), 16: 251-256.

[21] Souza DM, Rocha RF, Low caloric value of ethanol itself increases alveolar bone loss in ligature-induced periodontitis in male rats, Bras Oral Res (2009), 23: 460-466.

[22] Lamster IB, Oshrain RL, Celenti R, Levine K, Fine JB, Correlation analysis for clinical and gingival crevicular fluid parameters at anatomically related gingival sites, J Clin Periodontol (1991), 18: 272-277.

[23] Hagai Hazan-Molina, Abraham Z. Reznick, Hanna Kaufman,Dror Aizenbud, Assessment of IL- $1 \beta$ and VEGF concentration in a rat model during orthodontic tooth movement and extracorporeal shock wave therapy, Archives of Oral Biology 2012 October 22, [Epub ahead of print].

[24] Lalla E, Lamster IB, Feit M, Huang L, Schmidt AM, A murine model of accelerated periodontal disease in diabetes, J Periodontal Res (1998), 33: 387-399.

[25] de Lima V, Bezerra MM, de Menezes Alencar VB, Vidal FD, da Rocha FA, de Castro Brito GA, de Albuquerque Ribeiro $\mathrm{R}$, Effects of chlorpromazine on alveolar bone loss in experimental periodontal disease in rats, Eur J Oral Sci (2000), 108: 123-129.

[26] Carvalho RS, de Souza CM, Neves JC, Holanda-Pinto SA, 
Pinto LM, Brito GA, de Andrade GM, Effect of venlafaxine on bone loss associated with ligature-induced periodontitis in Wistar rats, J Negat Results Biomed (2010), 9: 3.

[27] Leitão RF, Ribeiro RA, Chaves HV, Rocha FA, Lima V, Brito GA, Nitric oxide synthase inhibition prevents alveolar bone resorption in experimental periodontitis in rats, $J$ Periodontol (2005), 76: 956-963.

[28] Steven Offenbacher, DDs, PhD, MMSC, 1 Silvana Barros, DDS, PhD, 1 L Mendoza, DDS, S Mauriello, MS, J Preisser, $\mathrm{PhD}, \mathrm{K}$ Moss, Marko de Jager, PhD, MMSc, 2 and Marcelo Aspiras, PhD2, Changes in Gingival Crevicular Fluid Inflammatory Mediator Levels during the Induction and Resolution of Experimental Gingivitis in Humans, $J$ Clin Periodontol (2010), 37: 324-333.

[29] Heasman PA, Collins JG, Offenbacher S, Changes in crevicular fluid levels of interleukin-1 beta, leukotriene B4, prostaglandin E2, thromboxane B2 and tumour necrosis factor alpha in experimental gingivitis in humans, $J$ Periodontal Res (1993), 28: 241-247.

[30] Beutler BA, Milsark IW, Cerami A, Cachectin/tumor necrosis factor: Production, distribution, and metabolic fate in vivo, $J$ Immunol (1985), 135: 3972-3977.

[31] Danchuk S, Ylostalo JH, Hossain F, Sorge R, Ramsey A, Bonvillain RW, Lasky JA, Bunnell BA, Welsh DA, Prockop DJ, Sullivan DE, Human multipotent stromal cells attenuate lipopolysaccharide-induced acute lung injury in mice via secretion of tumor necrosis factor- $\alpha$-induced protein 6 , Stem Cell Res Ther (2011), 2: 27.

[32] Bretz WA, Weyant RJ, Corby PM, Ren D, Weissfeld L, Kritchevsky SB, Harris T, Kurella M, Satterfield S, Visser M, Newman AB, Systemic inflammatory markers, periodontal diseases, and periodontal infections in an elderly popula- tion, J Am Geriatr Soc (2005), 53: 1532-1537.

[33] Spranger J, Kroke A, Möhlig M, Hoffmann K, Bergmann MM, Ristow M, Boeing H, Pfeiffer AF, Inflammatory cytokines and the risk to develop type 2 diabetes: Results of the prospective population-based European Prospective Investigation into Cancer and Nutrition (EPIC)-Potsdam Study, Diabetes (2003), 52: 812-817.

[34] Orbak R, Tezel A, Canakçi V, Demir T, The influence of smoking and non-insulin-dependent diabetes mellitus on periodontal disease, J Int Med Res (2002), 30: 116-125.

[35] Tracey K, Cerami A, Metabolic responses to cachectin/TNF, Annals of the New York academy of sciences (1990), 587: 325-331.

[36] Lalla E, Cheng B, Lal S, Kaplan S, Softness B, Greenberg E, Goland RS, Lamster IB, Diabetes mellitus promotes periodontal destruction in children, J Clin Periodontol (2007), 34: 294-298.

[37] Mishima N, Sahara N, Shirakawa M, Ozawa H, Effect of streptozotocin-induced diabetes mellitus on alveolar bone deposition in the rat, Arch Oral Biol (2002), 47: 843-849.

[38] Villarino ME, Sánchez LM, Bozal CB, Ubios AM, Influence of short-term diabetes on osteocytic lacunae of alveolar bone. A histomorphometric study, Acta Odontol Latinoam (2006), 19: 23-28.

[39] Santos Tunes R, Foss-Freitas MC, Nogueira-Filho Gda R, Impact of periodontitis on the diabetes-related inflammatory status, J Can Dent Assoc (2010), 76: a35.

[40] Nassar H, Kantarci A, van Dyke TE: Diabetic periodontitis, a model for activated innate immunity and impaired resolution of inflammation, Periodontol 2000 (2007), 43: 233-244.

[41] Akira S, Takeda K, Toll-like receptor signalling, Nat Rev Immunol (2004), 4: 499-511. 


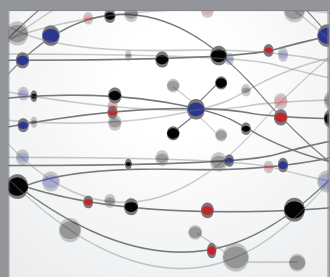

The Scientific World Journal
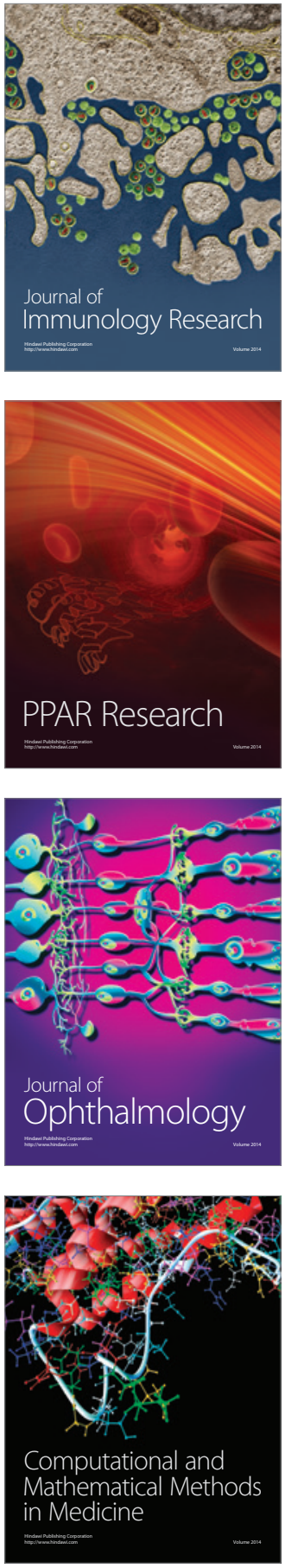

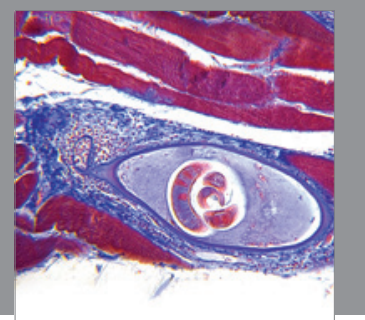

Gastroenterology

Research and Practice
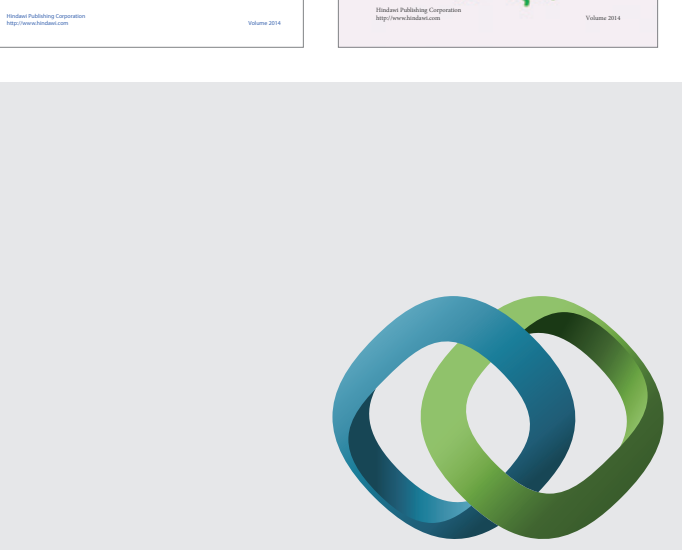

\section{Hindawi}

Submit your manuscripts at

http://www.hindawi.com
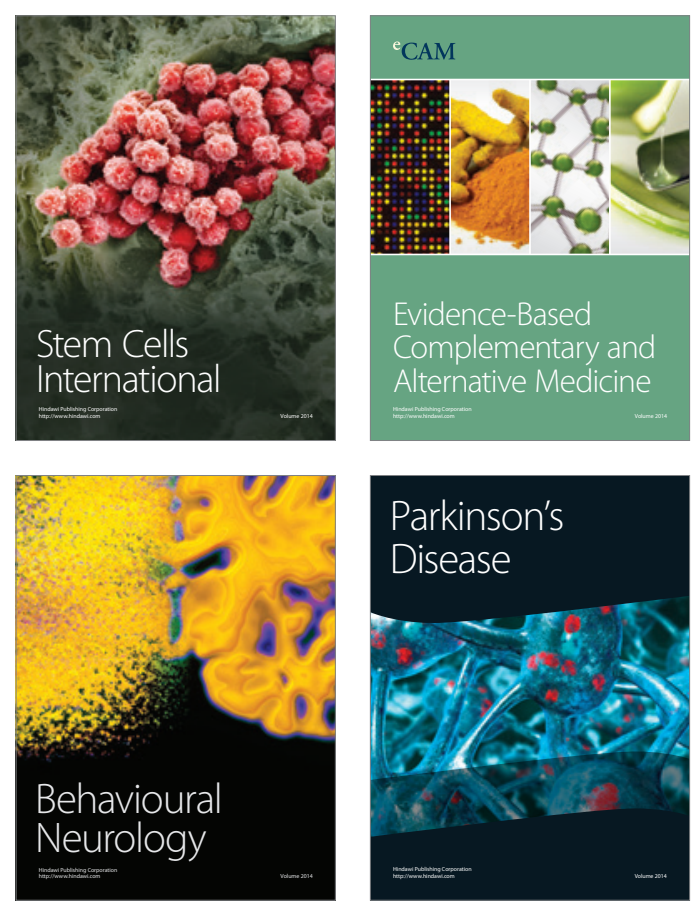

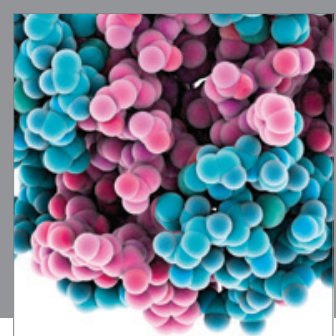

Journal of
Diabetes Research

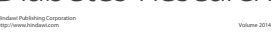

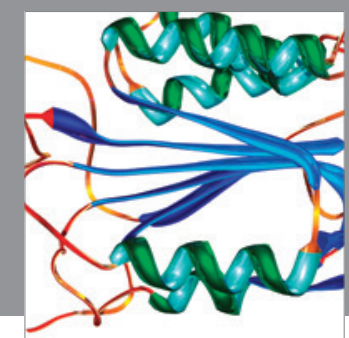

Disease Markers
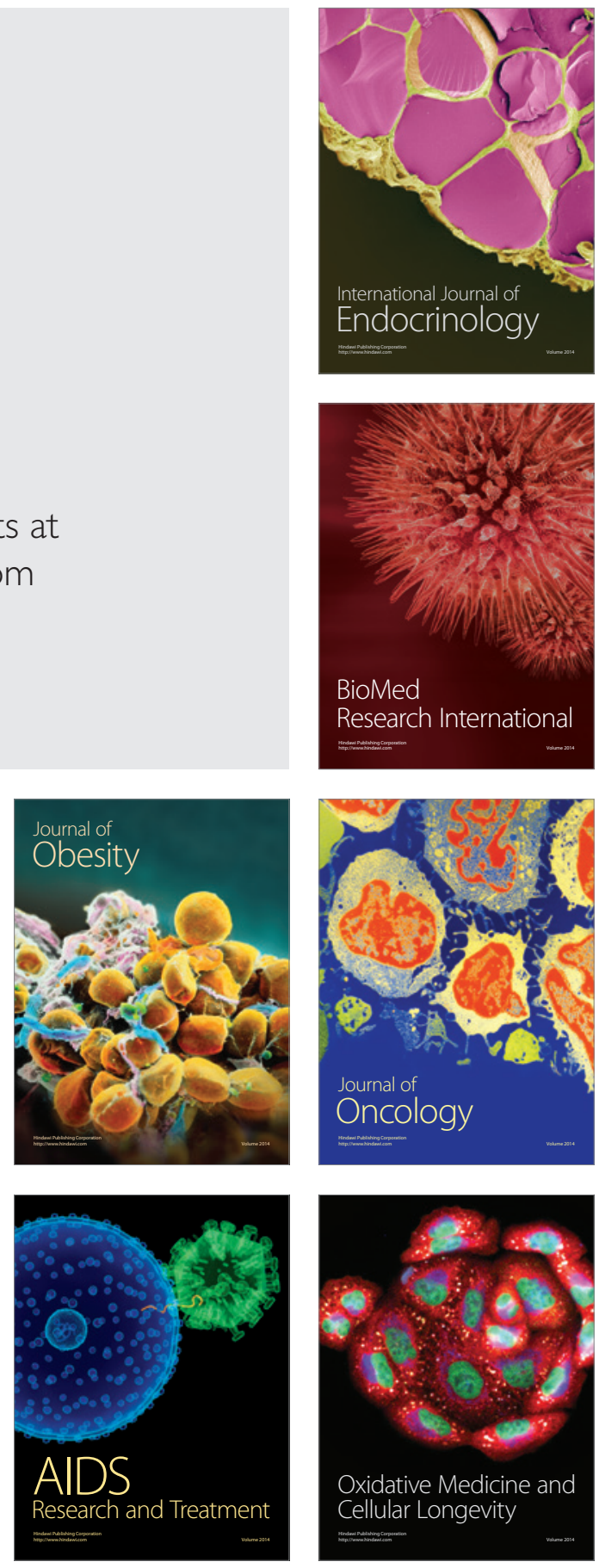